\title{
Oblique Transsphenoethmoidal Approach to the Cavernous Sinus
}

\author{
Živko GNJIDić, Tomislav SAJKO, Maša MALENICA, \\ Nenad KUdELIĆ, Jasna TALAN-HRANILOVIĆ* , and Zoran RUMBOLDT** \\ Department of Neurosurgery, and *“Ljudevit Jurak” University Department of Pathology, \\ "Sisters of Mercy" University Hospital, Zagreb, Croatia; \\ ** Department of Radiology, Medical University of South Carolina, \\ Charleston, South Carolina, U.S.A.
}

\begin{abstract}
Pituitary adenomas frequently invade the cavernous sinus. The standard transsphenoidal approach does not provide satisfactory visualization of the cavernous sinus structures. The transcranial approach has no advantages, and increases the operative trauma and complications. The oblique transsphenoethmoidal approach, a modified standard transsphenoidal approach, was used to treat 19 patients with pituitary adenomas invading the cavernous sinus. Complete tumor removal was achieved in 15 patients and subtotal removal in 4 patients. The patients tolerated this modified transsphenoethmoidal approach well and the postoperative results were satisfactory. Although the number of patients was too small to allow any statistical analysis, the results, compared with other series, are encouraging.
\end{abstract}

Key words: pituitary tumor, cavernous sinus invasion, neurosurgical approach

\section{Introduction}

Intrasellar pituitary adenoma is usually treated via a transsphenoidal approach, whereas pituitary adenoma with extrasellar spread is removed through a transcranial approach. However, the indications for transsphenoidal approach have expanded due to technological advancements and increased experience, and this approach is now the first choice for most pituitary adenomas. ${ }^{16,22)}$

Pituitary adenoma frequently invades the cavernous sinus, ${ }^{13)}$ by either infiltration or indentation. The border between the lateral wall of the sella and the medial wall of the cavernous sinus is formed by a thin, one-fold layer of the dura, which is commonly fenestrated..$^{825,28)}$ Indentation of pituitary adenoma into the cavernous sinus through large or small dural defects in the sellar lateral wall is a favorable indication for surgery. In contrast, infiltration of the cavernous sinus structures presents a difficult and complex problem, especially with hormonally active tumor. Removal of the largest portion of the tumor will resolve the space-occupying effect of the lesion, but a small residue of hormonally active tumor in the

\footnotetext{
Received April 7, 2005; Accepted June 14, 2008
}

cavernous sinus can continue to manifest as complex endocrine disease. Therefore, infiltration of the cavernous sinus presents some of the most difficult problems in pituitary surgery.

The standard transsphenoidal approach can treat pituitary adenoma with parasellar extension into the cavernous sinus region, as well as fair reduction of pituitary adenomas infiltrating the cavernous sinus. ${ }^{11)}$ However, the standard transsphenoidal approach does not provide satisfactory visualization of the cavernous sinus structures. The transcranial extradural or intradural approaches do not provide better visualization, and also increase the operative trauma and postoperative complications. ${ }^{2,9,10,14,27)}$ Recently, developments and refinements of endoscopic-assisted techniques have lead to use in the treatment of adenomas infiltrating the cavernous sinus, ${ }^{1,4)}$ but no larger series or long-term follow-up periods have been reported. ${ }^{24)}$ Remnant tumors may be resistant to medical therapy or the financial burden may be unacceptable. In Croatia, the gamma knife was unavailable for treating residual adenoma in the cavernous sinus, and final assessment of the results, especially in hormonally active tumors, can take to 2-3 years.

The sphenoid sinus is a cubic-shaped cavity in the 
sphenoid bone, closely related to the pituitary gland, optical nerves, cavernous sinus, and internal carotid artery (ICA), which form bulges on its walls. These structures may be visualized by removing the thin walls of the sphenoid sinus. The posterior ethmoidal cellulae narrow the approach to the superior and lateral parts of the sphenoid sinus in some cases. Opening and connecting the posterior ethmoidal cellula with the sphenoid sinus to form one single cavity allows very wide, direct visualization of the inferomedial part of the cavernous sinus. By doing so, removal of the tumor extension across the tuberculum sellae and the supradiaphragmal part is possible. ${ }^{18)}$

The present study describes our attempts to extend the conventional transsphenoidal approach into the oblique transsphenoethmoidal approach, based on the transmaxillosphenoidal approach for removing tumors invading the medial part of the cavernous sinus. ${ }^{3,12,15)}$

\section{Materials and Methods}

Planning of the transsphenoethmoidal approach was based on information given by computed tomography (CT) and magnetic resonance (MR) imaging, in particular the size and shape of the sphenoid and ethmoid sinuses, and their relationships with the sella turcica, the tumor, and the cavernous sinus. The relationship of the pituitary adenoma with the medial wall of the cavernous sinus was thoroughly examined to differentiate intracavernous impression (indentation) from invasion (infiltration). ${ }^{6,21,26)}$

We performed the transsphenoidal approach on 261 patients with tumors of the sellar region, 243 $(93 \%)$ pituitary adenomas and $18(7 \%)$ other tumors, between 1996 and 2001. The follow-up period ranged from 15 to 75 months. Radiological signs (grades II-IV, according to Knosp's classification) of parasellar expansion of the pituitary adenoma into the cavernous sinus were present in 62 patients $(25.5 \%) .{ }^{21)}$

The standard transsphenoidal approach was performed in 43 of these 62 patients. After the removal of the central intrasellar part of the tumor, the parasellar part of the tumor was removed using curved cochleae and aspirators under the control of microsurgical mirrors or endoscopy. Indentation of the cavernous sinus was found in 35 cases. Intraoperative macroscopic signs of invasion into the cavernous sinus, which were later histologically proven, were present in 8 cases.

The modified oblique transsphenoethmoidal approach was performed in the other 19 patients, 11 women and 8 men aged from 26 to 61 years (mean
43.9 years), who had neuroradiological signs of cavernous sinus invasion, 11 patients with grade III and 8 patients with grade IV according to Knosp's classification of parasellar expansion. Two patients had somatotropic adenomas, one patient had prolactinoma resistant to pharmacological therapy, and the other 16 patients had non-functioning adenomas. Some form of hypopituitarism was found in 14 patients. One patient had undergone transsphenoidal surgery on two previous occasions, and another patient had undergone both transsphenoidal and transcranial approaches.

\section{Technique}

The surgical procedure is performed under general endotracheal anesthesia. The patient is placed in a semi-sitting position with the thorax elevated at $30^{\circ}$ from the horizontal plane. The head is placed in a Mayfield head-holder in the anteroposterior direction or on a horse-shoe and rotated right to left at $30^{\circ}$ from the sagittal plane. A C-arm fluoroscope is prepared. Antiseptic solution (hexetidine or $1 \%$ povidone-iodine) is applied to the nose and mouth. The incision in the nasal mucosa is made on the contralateral side to the tumor, and is brought down to the nasal septum. A sublabial incision is exceptionally used for children or women with a small nose. The nasal incision is followed by subperichondrial and subperiosteal preparation of the septum. The cartilaginous septum is dislocated on the side of the tumor and the bony septum is resected up to the anterior wall of the sphenoid sinus.

Minimal maxillary osteotomy is performed with Kerrison punches and the nasal process of the maxilla is removed if needed. The Cloward asymmetrical speculum (Fig. 1) is introduced to expose the sphenoid. The anterior wall of the sphenoid sinus is opened and the sphenoid mucosa is removed. The longer tip of the asymmetrical speculum is placed in the sphenoid sinus, contralateral to the tumor. The shorter tip is placed on the side of the tumor so that its anterior margin reaches the anterior margin of the posterior ethmoidal cellula, which is then opened, with subsequent removal of the mucosa. By doing so, the connection between the sphenoid sinus and the ethmoid cellula appears as a single cavity (Fig. 2). Care must be taken during opening of the speculum to not damage the very thin medial wall of the orbit, which could result in grave complications. This maneuver offers direct visualization of the posterior and lateral walls of the sphenoid sinus, and is the key to opening the inferomedial part of the cavernous sinus.

The carotid bulge and the optic nerve bulge are 


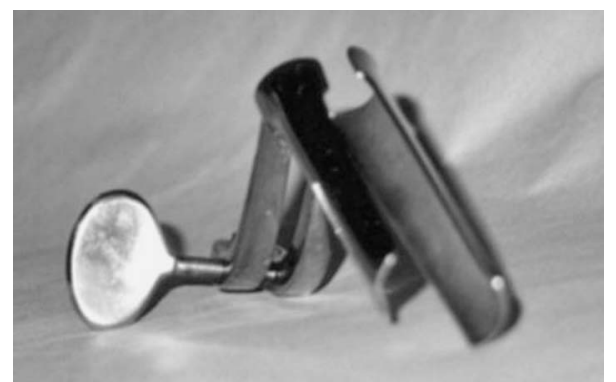

Fig. 1 Photograph showing the Cloward asymmetrical speculum.

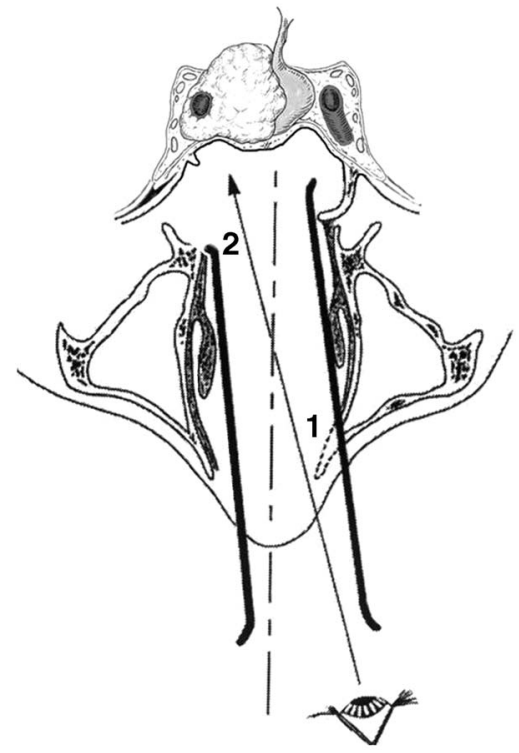

Fig. 2 Schematic representation of the oblique transsphenoethmoidal approach. Modified from Arita et al.3) Minimal maxillary osteotomy is performed (1). The asymmetrical speculum is then inserted, and the anterior wall of the sphenoid sinus is opened. The shorter tip is placed on the side of the tumor, and the posterior ethmoidal cellula is opened (2), creating a single cavity with the sphenoid sinus.

completely visible. An opening is made in the anterior wall of the sella and enlarged with small Kerrison punches to gain access to the anterior inferomedial part of the cavernous sinus. The tumor is usually seen protruding into the central part of the operative field, with no signs of extensive bleeding. Bleeding generally increases during tumor removal, but can be effectively and safely controlled with absorbable hemostatic material (oxidized regenerated cellulose). This wide approach allows complete removal of the intrasellar and intracavernous por-
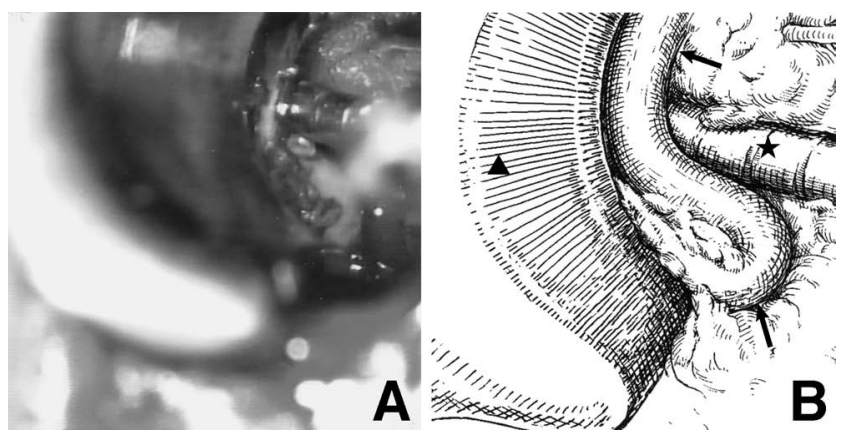

Fig. 3 Intraoperative photograph (A) and schematic drawing (B) showing the visualization of the internal carotid artery (ICA). The cavernous portion of the ICA is clearly seen (arrows), between the shorter tip of the speculum (triangle) and the suction tube (star).

tions of the tumor, and visualization of the intracavernous part of the ICA (Fig. 3). Residual dura forming the medial wall of cavernous sinus can be resected. The medial wall of the cavernous sinus shows changes in structure due to infiltration of invasive adenomas. Lateral from this line is the dura which represents the anterior wall of the cavernous sinus and separates it from the posterior and lateral sphenoid sinus wall. This part of the dura is often infiltrated by tumorous tissue which has been histologically proven in previous cases. The cranial nerves running through the lateral wall of the cavernous sinus remain intact. Any suprasellar extension of the tumor is also accessible. ${ }^{17,18)}$

After hemostasis, the tumor bed and the sphenoid sinus are loosely packed with adipose tissue, previously taken from the right thigh. We prefer adipose tissue over muscle due to the better contrast with the surrounding anatomical structures on postoperative CT or MR imaging. The anterior wall of the sella turcica is reconstructed using bony parts from the resected nasal septum. The entire region is then filled with fibrin glue. Tamponade of both nasal cavities is maintained for 4 days. After removal, a spray with isotonic seawater is locally applied.

\section{Results}

Complete removal of the tumor was achieved in 15 patients and subtotal removal in 4 patients. Two patients had very solid, adherent, fibrous adenomas that allowed removal of only the central intrasellar part with significant reduction of the intracavernous part, one somatotropic adenoma and one non-functioning adenoma. Biochemical markers in the patient with somatotropic adenoma after surgery 
(growth hormone, insulin-like growth factor-1) indicated residual adenoma. Postoperative irradiation was performed in both patients. The adenoma had expanded infratentorially through the cavernous sinus in the third patient, leaving a residual part that was treated also by radiotherapy. Subtotal removal of the pituitary adenoma was performed in the fourth patient due to frequent episodes of intraoperative bradycardia and extrasystolia.

Postoperatively, two patients developed transient partial anosmia on the side of the ethmoidectomy. Neuroophthalmologic evaluation revealed right abducens nerve palsy in one patient that resolved 2 months after the surgery. There were no other complications, and no deaths.

\section{Discussion}

In this series, 43 cases of parasellar expansion of pituitary adenomas were removed through the standard transsphenoidal approach. Visualization using microsurgical mirrors or a rigid sinoscope with a view angle of $45^{\circ}$ as sometimes helpful, but these methods are generally not suitable for such a delicate region. ${ }^{3)}$ Moreover, one hand of the surgeon was holding the mirror or sinoscope, which also reduced the already small working field in the speculum. Hence, removal of most tumors was performed without direct visualization of the intracavernous part of the tumor and intracavernous part of the ICA. Use of the endoscope with a view angle increases visibility of the parasellar space (cavernous sinus), but such an endoscope was not available at the time.

The oblique transsphenoethmoidal approach was used to treat 19 patients with pituitary tumors invading the medial part of the cavernous sinus. This approach allows complete visualization of the intracavernous part of the tumor and the intracavernous part of the ICA. The inferomedial approach to the cavernous sinus is extradural. One of the advantages is that tumor invading the medial part of the cavernous sinus is approached from the medial side, that is along the path of the tumor spread, so that the cranial nerves running through the lateral wall of the cavernous sinus remain intact. The risk of damaging cranial nerves using this approach is smaller than with the transcranial approach, as verified by the postoperative neurological status in this series (one patient with abducens nerve palsy, two patients with transient anosmia). ${ }^{7}$

The limiting factor is that mobilization of the ICA becomes necessary, causing bradycardia, in cases of tumor expansion lateral from the ICA. Signs of tumor invading the ICA were not found in any of our cases. This approach provides enough space for intraoperative clipping of the ICA, in case of rupture, but not to perform a bypass. Bleeding from the cavernous sinus was safely controlled using hemostatic material and compression. One possible complication is damage of the olfactory nerve during dissection of the nasal mucosa in the upper third of the nasal cavity. Another possible complication is a cerebrospinal fluid fistula from the cribriform plate, as a consequence of extensive nasal mucosa dissection.

Our series includes only 19 patients, which does not allow statistical analysis, but the comparison of our results with other studies is encouraging. ${ }^{5)}$ Similar oblique approaches achieved complete excision of tumors invading the cavernous sinus (Knosp grade III or IV) in 4 of $8^{12)}$ and 4 of $13^{3)}$ patients. Our results are significantly better, with complete excision in 15 of 19 patients (Fig. 4).

Unlike the ipsilateral transmaxillosphenoidal approach, ${ }^{12)}$ the contralateral oblique transsphenoethmoidal approach does not infringe the integrity of the maxillary sinus, and the minimal maxillary osteotomy in the inferolateral angle of the piriform aperture will not cause cosmetic or functional deficits in the viscerocranial region. The oblique transseptal approach and posterior submucosal ethmoidectomy maintains the nasal mucosa intact, so preventing direct communication between the nasal bacterial flora and the operative field. Although the nasal mucosa is not sterile, we did not apply antibiotics preoperatively. No inflammatory complications in the operative field occurred in any of the patients.

A specially constructed speculum allows easier manipulation. ${ }^{12)}$ Even more suitable specula have been constructed and recommended. ${ }^{3,19)}$ The space provided by the shorter tip of the nasal speculum allows exploration and removal of the intrasellar and intracavernous part of the tumor. ${ }^{23)}$ However, it is not necessary to purchase these asymmetrical retractors. When we first started using our approach, we were able to construct an asymmetrical retractor from the components of two standard Cloward retractors of various lengths.

Pituitary adenomas frequently invade the cavernous sinus in the medial to lateral direction, and structures running through the cavernous sinus may be laterally displaced. Modifications of transsphenoidal approach, such as the oblique transsphenoethmoidal approach, allow better visualization of the region, and more chance of achieving complete tumor removal, as well as minimizing the damage to vascular and neural structures in the cavernous sinus. ${ }^{20)}$ Endoscopic transsphenoidal sur- 

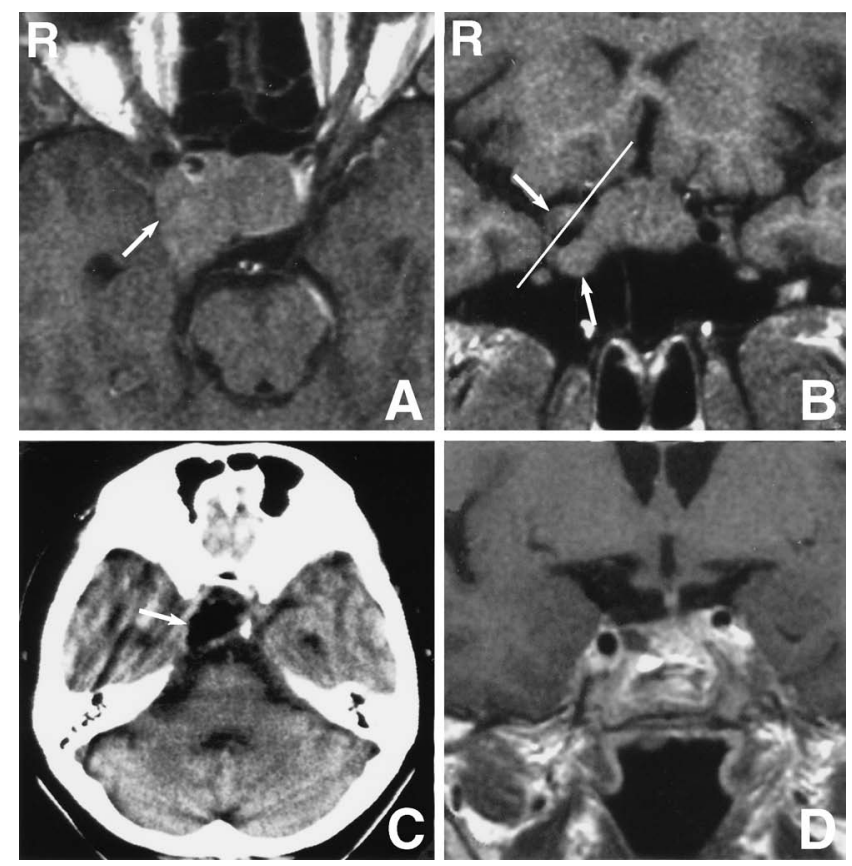

Fig. 4 A: Preoperative axial $\mathrm{T}_{1}$-weighted magnetic resonance (MR) image with contrast medium showing a pituitary mass extending to the right, lateral to the cavernous internal carotid artery (arrow). B: Preoperative coronal $T_{1}$-weighted $M R$ image with contrast medium revealing that the mass has probably invaded the right cavernous sinus (arrows). The tumor extends beyond the lateral intracavernous line (thin line), corresponding to grade IV according to Knosp's classification, and also in the medial venous sulcus (lower arrow). C: Postoperative axial computed tomography scan showing the fat packed in the sella and the right cavernous sinus (arrow). D: Postoperative coronal $T_{1}$-weighted $M R$ image with contrast medium showing no evidence of residual tumor.

gery will probably become the major method in pituitary surgery in the near future with present rapid development, but the oblique transsphenoethmoidal approach as a modified microscopic transsphenoidal approach will still be important in the transition period and in some special cases.

\section{References}

1) Alfieri A, Jho HD: Endoscopic endonasal cavernous sinus surgery: An anatomic study. Neurosurgery 48: 827-837, 2001

2) Al-Mefty O, Smith RR: Surgery of tumors invading the cavernous sinus. Surg Neurol 30: 370-381, 1988
3) Arita K, Kurisu K, Tominaga A, Ohba S, Ikawa F, Iida K, Yoshioka H: Transsphenoidal "cross court" approach using a slightly modified speculum to reach pituitary adenomas with lateral growth. Acta Neurochir (Wien) 142: 1055-1058, 2000

4) Cappabianca P, Alfieri A, Colao A, Ferone D, Lumbardy G, de Divitiis E: Endoscopic endonasal transsphenoidal approach: An additional reason in support of surgery in the management of pituitary lesions. Skull Base Surgery: An Interdisciplinary Approach 9: 109-117, 1999

5) Ciric I, Ragin A, Baumgartner C, Pierce D: Complications of transsphenoidal surgery: Results of a national survey, review of the literature, and personal experience. Neurosurgery 40: 225-237, 1997

6) Cottier JP, Destrieux C, Braunereau L, Bertrand P, Moreau L, Jan M, Herbreteau D: Cavernous sinus invasion by pituitary adenoma: MR imaging. Radiology 215: 463-469, 2000

7) Couldwell WT, Weiss MH, Rabb C, Liu JK, Apfelbaum RI, Fukushima T: Variations on the standard transsphenoidal approach to the sellar region, with emphasis on the extended approaches and parasellar approaches: surgical experiences in 105 cases. Neurosurgery 55: 547-550, 2004

8) Destrieux C, Kakou M, Velut S, Lefrancq T, Jan M: Microanatomy of the hypophyseal fossa boundaries. J Neurosurg 88: 743-752, 1998

9) Dolenc VV: Transcranial epidural approach to pituitary tumors extending beyond the sella. Neurosurgery 41: 542-552, 1997

10) Dolenc VV, Kregar T, Ferluga M, Fettich M, Morina A: Treatment of tumors invading the cavernous sinus, in Dolenc VV (ed): The Cavernous Sinus. A Multidisciplinary Approach to Vascular and Tumorous Lesions. New York, Springer-Verlag, 1987, pp 377-391

11) Falbusch R, Buchfelder M: Transsphenoidal surgery of parasellar pituitary adenomas. Acta Neurochir (Wien) 92: 93-99, 1988

12) Fraioli B, Esposito V, Santorio A, Lannetti G, Giuffre R, Cantore G: Transmaxillosphenoidal approach to tumors invading the medial compartment of the cavernous sinus. J Neurosurg 82: 63-69, 1995

13) Gnjidić Ž: [Evaluation of Clinical, Radiological and Laboratory Parameters in Acromegalic Patients Operated on by Transsphenoid Approach]. Zagreb, Croatia, University of Zagreb School of Medicine, 1994 (Cro) (thesis)

14) Hakuba A, Tanaka K, Suzuki T, Nishimura S: A combined orbitozygomatic infratemporal epidural and subdural approach for lesions involving the entire cavernous sinus. J Neurosurg 71: 699-704, 1989

15) Inoue $T$, Rhoton AL, Theele $D$, Barry ME: Surgical approaches to the cavernous sinus: A microsurgical study. Neurosurgery 26: 903-932, 1990

16) Jane JA, Thapar K, Kaptain GJ, Maartens N, Laws ER: Pituitary surgery: Transsphenoidal approach. Neurosurgery 51: 435-442, 2002

17) Kaptain GJ, Vincent DA, Sheehan JP, Laws ER: 
Transsphenoidal approaches for the extracapsular resection of midline suprasellar and anterior cranial base lesions. Neurosurgery 49: 94-101, 2001

18) Kato T, Sawamura $Y$, Abe H, Nagashima M: Transsphenoidal-transtuberculum sellae approach for supradiaphragmatic tumors: Technical note. Acta Neurochir (Wien) 140: 715-719, 1998

19) Kitano M, Taneda M: Extended transsphenoidal approach with submucosal posterior ethmoidectomy for parasellar tumors. J Neurosurg 94: 999-1004, 2001

20) Kitano M, Taneda M, Shimono T, Nakao Y: Extended transsphenoidal approach for surgical management of pituitary adenomas invading the cavernous sinus. J Neurosurg 108: 26-36, 2008

21) Knosp E, Steiner E, Kitz K, Matula C: Pituitary adenomas with invasion of the cavernous sinus space: A magnetic resonance imaging classification compared with surgical findings. Neurosurgery 33: 610-618, 1993

22) Laws ER: Transsphenoidal approach to pituitary tumors, in Schmidek HH, Sweet WH (eds): Operative Neurosurgical Techniques: Indications, Methods, and Results, ed 3. Philadelphia, WB Saunders, 1995, pp 283-292

23) Lüdecke D: Comments. Acta Neurochir (Wien) 142: 1058, 2000 (comment)

24) Lui J, Das K, Weiss M, laws E, Couldwell W: The history and evolution of transsphenoidal surgery. $J$ Neurosurg 95: 1083-1096, 2001

25) Romano A, Zuccarello M, van Loveren H, Keller JT: Expanding the boundaries of the transsphenoidal approach: a microanatomic study. Clin Anat 14: 1-9, 2001

26) Roux FX, Obreja C, Moussa R, Devaux B, Nataf F, Turak B, Page P, Meder JF: [Intracavernous extension of hypophyseal macroadenomas: infiltration or invagination?] Neurochirurgie 44: 344-351, 1998 (Fre, with Eng abstract)

27) Sekhar LN, Sen CN, Jho HD, Janecka I: Surgical treatment of intracavernous neoplasms: A four-year experience. Neurosurgery 24: 18-30, 1989

28) Yokoyama S, Hirano H, Moroki K, Goto M, Imamura S, Kuratsu J: Are nonfunctioning pituitary adenomas extending into the cavernous sinus aggressive and/or invasive? Neurosurgery 49: 857-863, 2001

Address reprint requests to: Živko Gnjidić, M.D., Department of Neurosurgery, "Sisters of Mercy" University Hospital, Vinogradska 29, Zagreb, HR-10000, Croatia.

e-mail: zgnjidic@kbsm.hr

\section{Commentary}

The authors reported a technical note of the oblique transsphenoethmoidal approach, a modified standard transsphenoidal approach, to treat 19 patients with pituitary adenomas invading the cavernous sinus. With minimal maxillary osteotomy and the use of a specially-designed asymmetrical Cloward speculum, they performed complete tumor removal in 15 patients.

I assume that this approach deserves to be noted in the current period when various approaches can be chosen to treat the pituitary adenoma extended into the cavernous sinus according to the surgeon's preference and the facilities of institutes.

This approach was developed by Fraioli et al., back in 1995.1) They had tried widening of the standard transsphenoidal approach by dislocation of the medial wall of maxillary sinus and the use of a custommade sphenoidal retractor with asymmetric blades, to inferomedially expose the prominences of bone above the carotid artery. Kitano et al. ${ }^{3)}$ reported the extended transsphenoidal approach with a submucosal posterior ethmoidectomy in the treatment of 36 patients with pituitary adenoma invading cavernous sinus. With those approaches, the inferior wall of the affected cavernous sinus was entirely exposed to permit safe removal of tumor as well as to secure the bony eminence portion of the ICA.

As the authors mentioned, however, the endoscopic transsphenoidal surgery which is now rapidly developing, will probably become the mainstay in the treatment of pituitary tumors with invasion into the cavernous sinus in near future. Jho et al. ${ }^{2)}$ had developed and reported an endoscopic endonasal approach to the cavernous sinus developed with cadaver study and subsequently used in patient treatment. With the development of fine surgical instruments for twohand surgery, better visualization and magnification technology, this endoscopic endonasal surgery will prevail in the neurosurgical practice for the patients with tumors in the skull base.

Kuo et al. ${ }^{4}$ reported the results of gamma knife radiosurgery for the treatment of 139 patients having benign cavernous sinus tumors with median follow-up of 3.5 years. They suggested that the gamma knife is a safe and effective treatment for selected patients with benign cavernous sinus tumors and is an important adjunct for treating residual and/or recurrent postoperative tumor.

Taken together, this oblique transsphenoethmoidal approach deserves to be applied in selected cases and during the current transitional period. 


\section{References}

1) Fraioli B, Esposito V, Santoro A, Iannetti G, Giuffre R, Cantore G: Transmaxillosphenoidal approach to tumors invading the medial compartment of the cavernous sinus. J Neurosurg 82: 63-69, 1995

2) Jho HD, Ha HG: Endoscopic endonasal skull base surgery: Part 2-The cavernous sinus. Minim Invasive Neurosurg 47: 9-15, 2004

3) Kitano M, Taneda M, Shimono T, Nakao Y: Extended transsphenoidal approach for surgical management of pituitary adenomas invading the cavernous sinus. J Neurosurg 108: 26-36, 2008

4) Kuo JS, Chen JC, Yu C, Zelman V, Giannotta SL, Petrovich Z, MacPherson D, Apuzzo ML: Gamma knife radiosurgery for benign cavernous sinus tumors: quantitative analysis of treatment outcomes. Neurosurgery 54: 1385-1394, 2004

Hee-Won JUNG, M.D. Department of Neurosurgery Seoul National University Hospital Seoul, R.O.K.

This is a very interesting paper, containing some important suggestions and concepts regarding the difficult problem of surgical approaches to tumors invading the cavernous sinus. The idea of using the asymmetrical Cloward retractor is a very good one, and the oblique approach is suggested seemed to be very effective in dealing with a variety of unusual tumor presentations. Despite the fact that it is rare that pituitary tumors actually surround the nerves in the cavernous sinus, it is important to reemphasize gentleness of technique in dissecting in this area.

Edward R. LAWS, Jr., M.D., F.A.C.S.
Department of Neurosurgery

Brigham \& Women's Hospital Boston, Massachusetts, U.S.A.

This is an important article suggesting a modified standard transsphenoidal approach to tumors that frequently invade the cavernous sinus. We have tried this technique a few times, encouraged by my mentor Prof. Jules Hardy from Montreal, after watching some of his cases. A few years ago Prof. Hardy was also enthusiastic about this approach, and modified his own speculum, in order to reach the parasellar expansions, following Dr. Fraioli's technique (ref. 12 of this article) and advice.

Dr. Hardy and myself have compared our results of cases operated by this oblique approach and reckoned that radical removal is not always possible, involving great risk in relation to the carotid arteries, mainly in bilateral cases; and in spite of our extensive statistics (over 4000 transsphenoidals) (personal communication) both of us still do not feel secure with this promising approach. Personally, in cases of Cushing's disease, I was forced to use Prof. Dolenc's technique plus radiotherapy in order to improve the symptoms in cases invading the cranial base (refs. 9 and 10 of this article).

I presume it is very difficult to speak on complete removal in these cases, if the degree of invasion is not reported and hormone levels are not followed in secreting adenomas.

Raul Marino, Jr., M.D., F.A.C.S. Professor-Emeritus of Neurosurgery University of São Paulo Medical School São Paulo, Brazil 